\title{
Adherencia al tamizaje de cáncer cérvicouterino: Una mirada desde el modelo de determinantes sociales de la salud
}

\author{
María Teresa Urrutia ${ }^{1 a}$, Macarena Gajardo. ${ }^{b}$ \\ ${ }^{1}$ Departamento de Salud de la Mujer. Escuela de Enfermería. Pontificia Universidad Católica de Chile. \\ a Enfermera Matrona, PhD. ${ }^{b}$ Alumna, Escuela de Medicina, Universidad de Chile.
}

Financiamiento: Proyecto financiado por CONICYT. FONDECYT 11130626.

\section{RESUMEN}

Antecedentes: Los Determinantes Sociales (DS) consideran "características del contexto social que afectan la salud, así como también los mecanismos por los cuales las condiciones sociales se traducen en impactos en salud". Las intervenciones cuyo objetivo son reducir la enfermedad serán exitosas solamente cuando consideren adecuadamente los DS de salud. Objetivo: Analizar, en base a la literatura existente, el modelo de determinantes sociales de la OMS y su aplicación a la adherencia al tamizaje de cáncer cérvicouterino (CC). Resultados: Son variados los estudios que establecen la relación entre los determinantes estructurales e intermediarios y la adherencia al tamizaje. Si bien hay estudios contradictorios, es relevante la asociación positiva entre determinantes estructurales y mayor adherencia: un mayor nivel educacional, la presencia de empleo y un mayor ingreso familiar determinan una mayor adherencia al tamizaje. Como determinantes estructurales, son varios los factores reportados, entre los que se encuentran las características del hogar, el tiempo de permanencia, las características del sistema de salud, su disponibilidad y contacto con profesionales de la salud, factores de riesgo para CC, conocimiento acerca de la enfermedad así como también las creencias, entre otras. Conclusión: Es necesario considerar la prevención de CC desde una perspectiva mucho más amplia, con un enfoque que va mas allá de los fenómenos biomédicos que la falta de adherencia al tamizaje conlleva, sino como una problemática social que explica dicho comportamiento en salud. Se sugiere que las campañas de prevención de CC consideren el enfoque de DS.

\section{PALABRAS CLAVE: Cáncer del cuello uterino, Papanicolaou, determinantes sociales de la salud}

\section{SUMMARY}

Background: Social Determinants consider "characteristics of the social context that affect health, as well as the mechanisms by which social conditions translate into health impacts". Interventions aimed at reducing diseases will be successful only when properly considered the social determinants of health. Objective: Analyze, based on the existing literature, WHO's Social Determinants of health model and its application to cervical cancer (CC) screening adherence. Results: There are many studies that establish the relationship between structural and intermediate determinants and adherence to screening. Even though there are contradictory studies, it is important the positive association between structural determinants and greater adherence: a higher education, employment and the presence of increased house holding come determine a greater adherence to screening. As structural determinants, several factors are reported, including house hold characteristics, residence time, characteristics of the health system, availability and contact with health professionals, risk factors for cervical cancer, knowledge and beliefs of the disease, 
among others. Conclusion: It is necessary to consider CC prevention from a much wider perspective, with an approach that goes beyond the biomedical phenomena that the lack of screening adherence entails, but as a social problem that explains this health behavior. It is suggested that CC prevention campaigns should consider the Social Determinants approach.

\section{KEY WORDS: Cervical cancer, Papanicolaou test, social determinants in health}

\section{INTRODUCCIÓN}

Los Determinantes Sociales (DS) consideran "características del contexto social que afectan la salud, así como también los mecanismos por los cuales las condiciones sociales se traducen en impactos en salud" $(1,2)$. El concepto de DS surge frente al reconocimiento de las limitaciones que tienen las intervenciones en salud, dirigidas a los riesgos individuales de enfermar, que no consideran el rol de la sociedad (1).

El enfoque de DS, plantea que la posición social determina la salud a través de factores intermediarios. El efecto en la salud es indirecto, mediante determinantes distribuidos de manera diferente en los grupos sociales (2).

Son tres los elementos centrales del modelo de DS de la OMS $(1,2)$ : 1 . Contexto social y político: el cual abarca múltiples elementos estructurales, culturales y funcionales de un sistema social, que resultan imposibles de medir a nivel individual; 2. Determinantes estructurales: aquellos que definen la posición socioeconómica; y 3. Determinantes intermedios: los cuales provienen de la estratificación social y determinan las diferencias en la exposición y vulnerabilidad de las personas. El sistema de atención de salud es un factor más y no la principal fuerza que determina la salud de los individuos (1).

Es sabido que la probabilidad de desarrollar cáncer, es mayor en las poblaciones socialmente más vulnerables, y que el riesgo de enfermar y morir está sistemáticamente asociado a menores niveles de ingresos y educación, y a mayores niveles de discriminación social (3).

Bajo este paradigma, la interrogante “¿Por qué algunas mujeres adhieren al tamizaje de Cáncer Cérvicouterino (CC) y otras no?", y por lo tanto la explicación de las bajas coberturas de Papanicolaou (PAP), podría resolverse a través de un enfoque de DS (4).

El objetivo del artículo es analizar, en base a la literatura, la adherencia al PAP bajo el modelo de DS de la OMS.

\section{METODOLOGÍA}

Se realizó una búsqueda bibliográfica en las bases de datos: CINHAL, Lilacs, ProQuest Medical Library, PsicINFO, Medline, Scielo y Scopus. Los descriptores fueron: adherencia y Papanicolaou, y sus correspondientes términos DECS/MESH en inglés, español y portugués. La búsqueda arrojó un total de 289 artículos de los cuales se seleccionaron aquellos que estudiaran factores relacionados a la adherencia a PAP.

El análisis de los determinantes se organizará según el modelo de DS, organizando la evidencia encontrada en determinantes estructurales e intermediarios y considerando la adherencia al tamizaje de CC como el resultado de salud esperado (Figura 1). Cabe señalar que ninguno de los artículos seleccionados analizó la adherencia al tamizaje desde esta perspectiva.

\section{DETERMINANTES ESTRUCTURALES (Tabla I)}

Nivel educacional. La literatura es consistente, señalando que la adherencia es mayor en aquellas mujeres que tienen más años de escolaridad (5-9). Cabe señalar y de manera paradójica que más de 16 años de educación no predice adherencia al tamizaje (8), lo que podría explicarse porque las mujeres con mayor educación tienen más responsabilidades, resultando en una falta de tiempo para adherir al tamizaje. Según Fernández y cols (9), la mayor adherencia se produce con 12 o más años de educación. Solo un estudio (10) no mostró asociación.

Ocupación. La ocupación de la mujer medida como presencia de empleo, ha sido señalada en diferentes investigaciones norteamericanas $(5,11)$ y europeas (12) relacionando una mayor adherencia al PAP con presencia de empleo. Sólo una investigación con mujeres asiáticas no mostró asociación (13), y una Investigacion con francesas (14) reportó lo contrario

Ingreso familiar. Es un DS relacionado al empleo u ocupación. Al respecto la literatura coincide en asociar un mayor ingreso familiar con una mejor adherencia $(5,7,11,13-23)$. Un estudio (20), donde se dividió a la población en tres grupos según ingreso, mostró que aquel con mayor ingreso adhería en un porcentaje significativamente mayor que los otros dos, incluso dividiéndolos según etnia. En una investigación (7) donde el ingreso económico no se asoció a la adherencia, el autor planteó la falta de asociación dado que es un examen sin costo. Una tesis doctoral realizada en mujeres afroamericanas en Estados Unidos tampoco reportó asociación (24). 


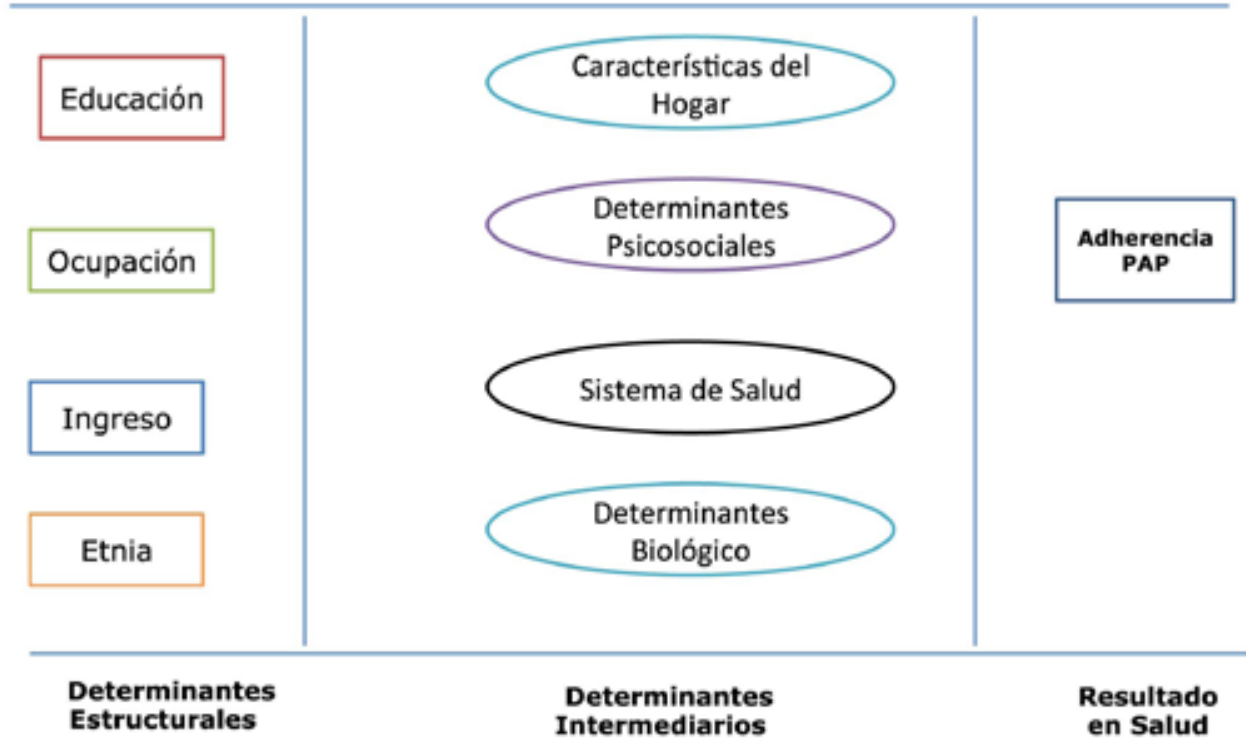

Figura 1. Determinantes sociales para la adherencia al tamizaje del cáncer cérvicouterino.

\section{Tabla I \\ DETERMINANTES ESTRUCTURALES PARA LA ADHERENCIA AL PAPANICOLAOU: RESULTADOS DE LAS INVESTIGACIONES}

\begin{tabular}{llcc}
\hline \multirow{2}{*}{$\begin{array}{l}\text { Determinantes } \\
\text { estructurales }\end{array}$} & \multicolumn{2}{c}{ Asociaciones encontradas } \\
\cline { 2 - 4 } Nivel educacional & Mayor adherencia al PAP & Menor adherencia al PAP & Sin asociación \\
\hline Ocupación & Mayor escolaridad & - & $\left(^{*}\right)$ \\
& $\begin{array}{l}\text { Menor desempleo } \\
\text { Mayor desempleo }\end{array}$ & - & $\left(^{*}\right)$ \\
Etnia & Mayor Ingreso & & $\left(^{*}\right)$ \\
& $\begin{array}{l}\text { Mejor adaptación cultural } \\
\text { Presencia de grupos étnicos } \\
\text { minorías }\end{array}$ & $\begin{array}{l}\text { Inmigrantes } \\
\text { Presencia de grupos étnicos } \\
\text { minorías }\end{array}$ & - \\
\end{tabular}

(*) Presencia de estudios que reportan no asociación entre la determinantes estructural y la adherencia al tamizaje de cáncer cérvicouterino.

Etnia. Ha sido considerada un DS que predice la adherencia al tamizaje de CC (19), fundamentalmente porque los antecedentes culturales de una mujer son un factor que afecta la búsqueda de atención médica (25). Cabe señalar que el efecto de la etnia en la adherencia podría explicarse por el bajo ingreso económico, falta de seguro de salud o mal manejo del idioma que estos grupos tienen (23). Un estudio holandés (20), que dividió a la población según lugar de nacimiento, mostró que las mujeres nacidas en Holanda adherían al tamizaje en un porcentaje significativamente mayor que las mujeres nacidas en otros países. El dominio del idioma ha sido la explicación dada en estudios con los mismos resultados (26), describiéndose que las mujeres más adaptadas a la cultura adhieren más al tamizaje, probablemente debido a que por su mejor manejo del idioma participan activamente en la sociedad y cuentan con mejor acceso a salud. Estos resultados hacen necesario considerar las poblaciones de inmigrantes al momento de intervenir por mejores coberturas de PAP (27). En 
Estados Unidos las mujeres latinas $(28,29)$ presentan una menor adherencia, sin embargo en Taiwán, las mujeres pertenecientes a minorías raciales son las que presentan mayor adherencia (30). Por otro lado, Cook y cols (31), señalan que las mujeres pertenecientes a minorías étnicas adhieren más al tamizaje del CC, llegando a ser hasta el doble la adherencia de las mujeres latinas y afroamericanas viviendo en USA, en comparación con las mujeres blancas, incluso ajustando estos resultados a variables como la edad y el seguro de salud.

\section{DETERMINANTES INTERMEDIARIOS (Tabla II)}

Características del hogar. La ubicación de la vivienda (urbano-rural) se ha relacionado a la adherencia al tamizaje, señalando que las mujeres que viven fuera de la ciudad adhieren menos $(8,10,32)$. El acceso limitado a la atención sanitaria, debido a la poca cantidad de médicos y centros de salud existentes en estas zonas, podría ser la explicación de este hallazgo. Sin embargo Challier y cols (14), reportan una mejor adherencia al disminuir la densidad de población.

Otra característica del hogar a considerar es el número de habitantes, donde una mejor adherencia se da en hogares con menos habitantes (14). Una mayor adherencia al tamizaje ha sido también asociada a una menor cantidad de hogares arrendados (14), lo que en parte estaría reflejando la ocupación y el ingreso de la mujer y su grupo familiar.

En relación al tiempo de permanencia de las personas en un determinado país o ciudad, un estudio con mujeres asiáticas en Estados Unidos (19) reveló que una permanencia en el país menor a 10 años se asocia a menor adherencia, relacionando este hallazgo con el manejo del idioma inglés. Al comparar los programas de tamizaje en Canadá y USA (33), las mujeres inmigrantes presentan menor adherencia que las nativas en ambos países. Challier y cols (14), señalan una mejor adherencia en sectores con menor cantidad de personas que se cambian de domicilio.

Sistema de salud. La presencia de seguro de salud es un factor que determina una mayor adherencia al tamizaje $(6,8,11,13,19,22,31,32,34-36)$. En el estudio de Cook y cols (31) dicha asociación varió según la edad, siendo mayor en mujeres menores de 45 años. Solo en un estudio realizado en mujeres latinas en USA (23), el seguro de salud no fue significativa su asociación.
Otro determinante que se considera positivamente asociado a la adherencia es el contacto con un servicio de salud o profesional de salud (37). Un mayor número de visitas médicas $(5,15)$, la presencia de un control médico en el último tiempo $(10,12$, $21,35,38,39)$ y contar con prestador de salud habitual $(33,40)$ son predictores de mayor adherencia. Específicamente, se ha descrito que visitar al mismo ginecólogo también se considera como un factor de adherencia importante; esto puede deberse al efecto que genera una mejor relación profesional y un mejor seguimiento de la mujer (10).

La disponibilidad de servicios médicos (14) señala una mayor adherencia en zonas con mayor número de profesionales y de servicios de salud. La intervención específica de un profesional de la salud (23) es predictor de mayor adherencia (34), lo que probablemente se relaciona con la indicación de tamizaje que el profesional hace a la mujer. La adherencia a otros tamizajes como la mamografía $(6,10,23,36,37,41)$, auto examen de mama $(23,36,41)$, examen de HIV y de hepatitis C (12) han sido considerados determinantes de mayor adherencia al PAP.

Determinantes psicosociales. En relación a la presencia de pareja, el resultado más frecuentemente reportado es que el matrimonio $(6,12,23,27,30,34,40,42)$ o la presencia de pareja $(16,18,19,38)$ determina una mayor adherencia, lo que probablemente estaría explicado porque la presencia de pareja requiere la utilización de algún método de planificación familiar (40) lo que llevaría a la mujer a consultar al ginecólogo, exponiéndose a la toma del PAP. El mismo estudio propone que el estigma asociado a la actividad sexual extramarital podría disuadir a las mujeres solteras de someterse al tamizaje de CC. Solo un estudio reporta que las mujeres adherentes son menos propensas a ser casadas o tener pareja (5). Eaker y cols (10), no encontraron asociación entre ambas variables.

El conocimiento y las creencias que la mujer tiene acerca del PAP y del CC han sido motivo de múltiples investigaciones, reportándose que una mejor adherencia se produce en mujeres con mayor conocimiento $(10,23,17,30,34,35,40,43)$, y con mejores (correctas) creencias $(10,12,13,17,27,40,44)$. Dentro de las principales razones que impiden una mejor adherencia son el miedo o vergüenza de ir al médico (42). La falta de conocimiento y el miedo al ridículo son importantes barreras para adherir al tamizaje, así como también la preocupación por el dolor que pudiera causar el examen (40). 


\section{Tabla II \\ DETERMINANTES INTERMEDIARIOS PARA LA ADHERENCIA AL PAPANICOLAOU: RESULTADOS DE LAS INVESTIGACIONES}

\begin{tabular}{|c|c|c|c|c|}
\hline \multicolumn{2}{|c|}{ Determinantes intermediarios } & \multicolumn{3}{|c|}{ Asociaciones encontradas } \\
\hline & & & & \\
\hline \multirow{4}{*}{$\begin{array}{l}\text { Características } \\
\text { del hogar }\end{array}$} & $\begin{array}{l}\text { Ubicación de la } \\
\text { vivienda }\end{array}$ & Menor densidad de población & Fuera de la ciudad & - \\
\hline & $\begin{array}{l}\text { Número de } \\
\text { habitantes }\end{array}$ & Menor número de habitantes & - & - \\
\hline & $\begin{array}{l}\text { Arriendo de la } \\
\text { Vivienda }\end{array}$ & $\begin{array}{l}\text { Menor cantidad de viviendas } \\
\text { arrendadas }\end{array}$ & - & - \\
\hline & $\begin{array}{l}\text { Tiempo de } \\
\text { permanencia }\end{array}$ & $\begin{array}{l}\text { Menor cantidad de personas } \\
\text { que cambian de domicilio }\end{array}$ & $\begin{array}{l}\text { Menor a } 10 \text { años en el país } \\
\text { Mujeres inmigrantes }\end{array}$ & - \\
\hline \multirow{4}{*}{ Sistema de salud } & Seguro de salud & Presencia de seguro de salud & - & $\left({ }^{\star}\right)$ \\
\hline & $\begin{array}{l}\text { Contacto con } \\
\text { servicio de salud }\end{array}$ & $\begin{array}{l}\text { Mayor número de visitas al } \\
\text { centro de salud } \\
\text { Presencia de un control } \\
\text { médico en el último tiempo } \\
\text { Presencia de un prestador de } \\
\text { salud habitual } \\
\text { Visitar al mismo ginecólogo }\end{array}$ & - & - \\
\hline & $\begin{array}{l}\text { Disponibilidad de } \\
\text { servicios médicos }\end{array}$ & $\begin{array}{l}\text { Mayor número de profesionales } \\
\text { Mayor número de servicios de } \\
\text { salud }\end{array}$ & - & - \\
\hline & $\begin{array}{l}\text { Otros tamizajes o } \\
\text { exámenes }\end{array}$ & $\begin{array}{l}\text { Mayor adherencia a otros } \\
\text { tamizajes o exámenes. }\end{array}$ & - & - \\
\hline \multirow[b]{2}{*}{$\begin{array}{l}\text { Determinantes } \\
\text { psicosociales }\end{array}$} & Presencia de pareja & Mujer casada o con pareja & Mujer casada o con pareja & $\left({ }^{\star}\right)$ \\
\hline & $\begin{array}{l}\text { Creencias } \\
\text { Conocimiento } \\
\text { Autoeficacia }\end{array}$ & $\begin{array}{l}\text { Mayor conocimiento } \\
\text { Mejores creencias } \\
\text { Mayor autoeficacia }\end{array}$ & - & - \\
\hline \multirow{3}{*}{$\begin{array}{l}\text { Determinantes } \\
\text { biológicos }\end{array}$} & Edad & $\begin{array}{l}\text { Menor edad } \\
\text { Mayor edad }\end{array}$ & $\begin{array}{l}\text { Mayor edad } \\
\text { Inmigrantes de mayor edad } \\
\text { Edad de migración mayor }\end{array}$ & - \\
\hline & $\begin{array}{l}\text { Enfermedades } \\
\text { crónicas y } \\
\text { discapacidad }\end{array}$ & $\begin{array}{l}\text { Menor cantidad de } \\
\text { enfermedades crónicas } \\
\text { Menor discapacidad }\end{array}$ & - & - \\
\hline & $\begin{array}{l}\text { Antecedente de } \\
\text { cáncer }\end{array}$ & $\begin{array}{l}\text { Antecedente personal de } \\
\text { cáncer } \\
\text { Antecedente familiar de cáncer }\end{array}$ & - & - \\
\hline \multirow{5}{*}{$\begin{array}{l}\text { Determinantes } \\
\text { biológicos: } \\
\text { factores de riesgo } \\
\text { para CC }\end{array}$} & $\begin{array}{l}\text { Número de factores } \\
\text { de riesgo }\end{array}$ & - & $\begin{array}{l}\text { Mayor numero de factores } \\
\text { de riesgo }\end{array}$ & - \\
\hline & $\begin{array}{l}\text { Edad de la primera } \\
\text { relación }\end{array}$ & - & Menor edad & $\left({ }^{\star}\right)$ \\
\hline & $\begin{array}{l}\text { Número de parejas } \\
\text { sexuales }\end{array}$ & $\begin{array}{l}\text { Más de una pareja en el último } \\
\text { año }\end{array}$ & $\begin{array}{l}\text { Dos o más parejas en los } \\
\text { últimos cinco años }\end{array}$ & - \\
\hline & Hábito tabáquico & $\begin{array}{l}\text { Mujeres que no fuman } \\
\text { Mujeres que han dejado de } \\
\text { fumar }\end{array}$ & - & - \\
\hline & $\begin{array}{l}\text { Resultado de PAP } \\
\text { previos }\end{array}$ & PAP alterado & PAP alterado & - \\
\hline
\end{tabular}




\section{CONTINUACIÓN TABLA II}

\begin{tabular}{|c|c|c|c|c|}
\hline \multicolumn{2}{|c|}{ Determinantes intermediarios } & \multicolumn{3}{|c|}{ Asociaciones encontradas } \\
\hline & & $\begin{array}{c}\text { Mayor adherencia } \\
\text { al PAP }\end{array}$ & $\begin{array}{c}\text { Menor adherencia } \\
\text { al PAP }\end{array}$ & $\begin{array}{c}\text { Sin } \\
\text { asociación }\end{array}$ \\
\hline \multirow{4}{*}{$\begin{array}{l}\text { Determinantes } \\
\text { biológicos: } \\
\text { características del } \\
\text { embarazo y parto, } \\
\text { planificación } \\
\text { familiar }\end{array}$} & Presencia de hijos & $\begin{array}{l}\text { Bajo número de niños } \\
\text { pequeños (menor a } 5 \text { años) }\end{array}$ & Sin hijos & $\left({ }^{*}\right)$ \\
\hline & $\begin{array}{l}\text { Número de } \\
\text { embarazos }\end{array}$ & Cuatro o más embarazos & - & - \\
\hline & Edad primer parto & Mujer joven & $\begin{array}{l}\text { Mujer joven } \\
\text { Parto después de los } 25 \\
\text { años }\end{array}$ & - \\
\hline & $\begin{array}{l}\text { Método de } \\
\text { planificación familiar }\end{array}$ & Uso de anticonceptivo oral & Uso de condón & - \\
\hline
\end{tabular}

(*) Presencia de estudios que reportan no asociación entre la determinante estructural y la adherencia al tamizaje de cáncer cérvicouterino.

Relacionado a las creencias, se encuentra la percepción de riesgo o susceptibilidad que la mujer perciba, encontrándose que el no considerarse en riesgo de desarrollar $\mathrm{CC}$ es motivo de baja adherencia (42). Asociado a esto se encuentra la creencia que el PAP requiere ser tomado cuando se presentan síntomas (45). A lo anterior se agrega un gran porcentaje de mujeres que cree que el PAP detecta un CC ya establecido (10). Esta situación hace necesario mejorar la información sobre el propósito del PAP, disminuyendo la ansiedad que este examen pueda producir. Respecto de la autoeficacia (46) definida como la convicción personal de poder realizar con éxito una conducta requerida que produzca resultados, también ha sido considerada una variable asociada a la adherencia al PAP (47-51).

Determinantes biológicos. La edad es otro factor descrito como predictor para adherir al tamizaje, siendo muchas veces señalado como el único determinante biológico. Al respecto la literatura es contradictoria. Al comparar grupos de mujeres de diferentes rangos etarios, a menor edad mayor adherencia al PAP $(5,11,12,14,18-23,27-29,31$ $33,37,43,52)$, sin embargo algunas investigación señalan lo contrario $(13,15,53)$. Se plantea que la edad es un factor que influye en la integración cultural de mujeres inmigrantes (27), siendo mejor en las mujeres jóvenes, quienes buscan integrarse al sistema de salud por temas de planificación familiar, adoptando conductas preventivas y por lo tanto mejorando la adherencia. En este mismo contexto, las mujeres inmigrantes de mayor edad adhieren menos debido a que enfrentan mayores barreras en el sistema de salud y también porque las reco- mendaciones médicas sobre la frecuencia del tamizaje se vuelven menos insistentes. Se ha sugerido también que las mujeres mayores adhieren menos debido a que están poco acostumbradas o se avergüenzan más de tomarse el examen (14). En un estudio de inmigrantes en Estados Unidos, la edad al momento de llegar al país fue determinante de adherencia al tamizaje (34), siendo peor la adherencia mientras mayor es la mujer al momento de migrar.

En relación a la presencia de enfermedades crónicas, las mujeres que adhieren al tamizaje presentan menor cantidad de enfermedades crónicas (5), así como también menor proporción de discapacidad (54). En este último estudio, al analizar los diferentes niveles de discapacidad, aquellas con discapacidad severa presentaron la menor adherencia. Al respecto se propusieron dos posibles explicaciones: la idea errónea que podrían tener los profesionales de salud que las mujeres discapacitadas son "asexuadas" y por lo tanto presentan menor riesgo de desarrollar CC y la dificultad para poder realizar el PAP.

En relación al antecedente familiar de cáncer, la adherencia se vería favorecida en aquellas mujeres con antecedentes personales o familiares de cáncer $(8,23)$. En un estudio realizado en Brasil, los autores reportan una menor adherencia en mujeres con mayor cantidad de factores de riesgo para desarrollar CC (18). Al respecto la edad de la primera relación sexual fue menor en el grupo de mujeres que no adhieren (33). En un estudio sueco (10), no se encontró asociación. El número de parejas sexuales ha sido también relacionado a la adherencia, señalando que las mujeres con más de una 
pareja en el último año adhieren más (12), y que la presencia de 2 o más parejas sexuales en los últimos 5 años genera una menor adherencia (10).

El hábito tabáquico, otro factor de riesgo para $\mathrm{CC}$, señala que la adherencia mejora en mujeres que no fuman $(10,28,32,33,35)$ o que han dejado el hábito $(15,45)$. Se propone como explicación que por un lado el cese del hábito tabáquico produciría un impacto positivo en la salud general de la mujer y por otro que los profesionales de la salud, al tener contacto con las mujeres que adhieren, aconsejan dejar el hábito tabáquico. En un estudio coreano (7) se vio que las mujeres que no estaban dispuestas o eran incapaces de dejar de fumar, eran más propensas a no adherir a cualquier tipo de tamizaje en general. La adherencia también ha sido asociada a los resultados de PAP previos, siendo en algunos casos la presencia de un PAP alterado un predictor de mayor (39) o menor adherencia (28).

Los antecedentes obstétricos también han sido relacionados, encontrándose que a menor número de niños pequeños (de 5 años o menos) es mejor la adherencia (14). En mujeres que no han tenido hijos se ha reportado menor adherencia $(38,42)$. Dos estudios señalan no asociación con la variable hijos $(10,24)$. En relación al número de embarazos la presencia de 4 o más embarazos es un predictor de adherencia en mujeres coreanas (7), mientras que un estudio en Grecia reportó lo contrario (8), postulando que a mayor número de embarazos se vuelve peor la adherencia. La edad al primer parto también ha sido estudiada, encontrándose ambas posibilidades: las mujeres más jóvenes son más adherentes (7) o lo contrario (17). En Brasil (18) las mujeres cuyo parto fue después de los 25 adhieren menos al tamizaje.

Por otro lado, la presencia de método anticonceptivo oral predice mejor adherencia $(5,10,17,43)$, lo contrario ocurre en mujeres usuarias de condón (10). Se planteó que el hecho de usar protección para evitar la transmisión de infecciones de transmisión sexual podría llevar a una menor búsqueda de atención médica.

\section{DISCUSIÓN}

Al momento de analizar los diferentes factores es importante considerar las propias definiciones que el modelo de DS señala: 1) cada factor es importante por si mismo, sin embargo al estar interrelacionados la influencia combinada determina el estado de salud, 2) actúan a diferentes niveles, 3) son acumulativos, 4) son causales (directa o indirectamente) o protectores y, 5) su actuar puede ser bidireccional. Dada la complejidad de las categorías sociales, es necesario clarificar que no se puede hablar de causa en el sentido clásico, por lo que bajo este paradigma es necesario hablar de explicaciones y de cómo dichas explicaciones se vinculan con un efecto, mediante una relación de "determinación" (4).

La cobertura de PAP a nivel nacional ha disminuido paulatinamente en los últimos años, desde un $67 \%$ en el año 2008 hasta un $59 \%$ en el 2011 (55). Diferentes campañas en Chile han sido implementadas en los últimos años con el objetivo dealcanzar la cobertura de $80 \%$, sin embargo ninguna de ellas ha tenido éxito. En el año 2007 fue lanzada la campaña nacional contra el CC "Pasa la Voz", una iniciativa educativa que buscaba concientizar a la población femenina sobre la existencia de esta enfermedad, la importancia de su detección y prevención a través de la realización del PAP. Dicha campaña buscaba convertirse en una cadena de comunicación en el que cada mujer transmitía a través de sus redes de contacto información sobre el $\mathrm{CC}$, a fin de generar conciencia de prevención y de detección precoz (56).

La minuta de la última campaña del MINSAL en el 2012, señala que el descenso paulatino de la cobertura a nivel nacional es "preocupante", para lo cual se presenta como estrategia una campaña de comunicación para lograr revertir el descenso en la cobertura. Dicha campaña establece seis mensajes, cuatro de los cuales invitan a difundir a nivel individual información acerca del examen (población objetivo, gratuidad, detección precoz, cobertura AUGE), el quinto mensaje invita a acudir al centro de salud más cercano y el último de ellos a focalizar la campaña en población de riesgo.

Si se analiza el foco de ambas campañas y de otras realizadas previamente, este ha estado dirigido a nivel estrictamente individual, asumiendo que la no adherencia de la mujer al tamizaje es un resultado de la falta de conocimiento acerca del examen o de la enfermedad.

Por lo anterior sugerimos que las campañas de prevención de CC consideren el enfoque de DS, dejando la no adherencia al tamizaje como resultado sólo de la falta de conocimiento. Si bien muchas de las campañas han sido elaboradas con un enfoque de riesgo, sólo han considerado el "riesgo de enfermar de CC" sin considerar el "riesgo de no adherir al tamizaje". Por otro lado es necesario considerar campañas que incluyan no tan solo variables biomédicas (como son los factores de riesgo para desarrollar la enfermedad) sino también variables no biomédicas que pudieran influir en la decisión de la mujer de adherir o no al tamizaje, como las analizadas en este artículo.

\section{CONCLUSIÓN}

Es necesario considerar la prevención de CC desde una perspectiva mucho más amplia, con un enfoque que va mas allá de los fenómenos biomédicos que la falta de adherencia al tamizaje conlleva, sino como una problemática social que explica 
dicho comportamiento en salud. Se requieren investigaciones que consideren los diferentes factores o determinantes, y sus respectivas interrelaciones en grupos de mujeres que adhieren y que no adhieren al tamizaje.

\section{REFERENCIAS}

1. Frenz P. Desafíos en Salud Pública de la Reforma: Equidad y Determinantes Sociales de la Salud. Rev Chil Salud Pública 2005;9(2):103-10.

2. World Health Organization. A conceptual framework for action on the social determinants of health. 2010. Citado 20 Abril 2013. Disponible en: http://www.who. int/sdhconference/resources/ConceptualframeworkforactiononSDH_eng.pdf.

3. Moiso A. Determinantes de la Salud. En: Fundamentos de Salud Pública. Barragan H. Citado 29 Abril 2013. Disponible en http://www.inus.org.ar/documentacion/Documentos\%20Tecnicos/Fundamentos_de_ la_salud_publica/cap_6.pdf.

4. Arias S. Inequidad y Cáncer: una revisión conceptual. Rev Nac Salud Publica 2009;27(3):342-48.

5. Worthington C, McLeish K, Fuller-Thomson E. Adherence over time to cervical cancer screening guidelines: Insights from the Canadian National Population Health Survey. J WomensHealth 2012;21(2):199-208.

6. Amonkar M, Madhavan S. Compliance rates and predictors of cancer screening recommendations among Appalachian women. J Health Care Poor Underserved 2002;13(4):443-60.

7. Lee M, Sun Chang H, Park EU, Yu SH, Yu SH, Sohn $\mathrm{M}$, Lee $\mathrm{S}$. Factors associated with participation in Korean women in cervical cancer screening examination by age group. Asian Pac J Cancer Prev 2011;12(6):1457-62.

8. Simou E, Foundoulakis E, Kourlaba G, Maniadakis N. Factors associated with the use of preventive services by women in Greece. Eur J Public Health 2010;21(4): 512-19.

9. Fernandez M, Lin J, Leong-Wu C, Aday L. Pap smear screening among Asian Pacific Islander women in a multisite community-based cancer screening program. Health Promot Pract 2009;10(2):210-21.

10. Eaker $S$, Adami $H$, Sparén $P$. Reasons women do not attend screening for cervical cancer: a populationbased study in Sweden. Prev Med 2001;32(6):482-91.

11. Reiter $P$, Linnan L. Cancer screening behaviors of African American Women enrolled in a communitybased cancer prevention trial. J Womens Health 2011;20(3):429-4 38.

12. Grange G, Malvy D, Lancon F, Gaudin AF, ElHasnaoui $A$. Factors associated with regular cervical cancer screening. Int J Gynaecol Obstet 2008;102(1):2833.

13. Ma G, Toubbeh J, Wang M, Shive S, Cooper L, Pham A. Factors associated with cervical cancer screening compliance and non compliance among Chinese, Korean, Vietnamese, and Cambodian Women. J Natl Med Assoc 2009;101(6):541-51.

14. Challier B, Meslans Y, Viel J. Deprived areas and attendance to screening of cervix uteri cancer in a French region. Cancer Causes Control 2000;11(2):157-62.

15. Maryinez-Huedo MA, Lopez de Andres A, HernandezBarrera V, Carrasco Garrido P, Martinez D, JimenezGarcia R. Adherence to breast and cervical cancer screening in Spanish women with diabetes: Associated factors and trend between 2006 and 2010. Diabetes Metab 2012;38:142-8.

16. Paskett E, Phillips K, Miller M. Improving compliance among women with abnormal Papanicolaou smears. ObstetGynecol 1995;86(3):353-9.

17. Wall K, Nuñez G, Salinas-Martínez A, Baraniuk S, Day RS. Modifiable barriers to cervical cancer screening adherence among working women in Mexico. $\mathrm{J}$ Womens Health 2010;19(7):1263-70.

18. Cesar J, Horta B, Gomes G, Houlthausen R, Willrich $R$, Kaercher A, lastrenski F. Factors associated with non-participation in screening for cervical cancer in Southern Brazil. Cad Saude Publica 2003;19(5):136572.

19. Lin M, Moskowitz J, Kazinets G, Ivey S, Kim YB, McDonell D. Adherence to Pap test guidelines: Variation among Asians in Califormia. Ethn Dis 2009;19:42532.

20. Van Leeuwen A, de Nooijer P, Hop W. Screening for cervical carcinoma. Participation and results for ethnic groups and socioeconomic status. Cancer Cytopathol 2005;105:270-6.

21. Randolph W, Freeman D, Freeman J. Pap smear use in a population of older Mexican-American women. Women Health 2002;36(1):21-31.

22. Jennings-Dozier K, Lawrence D. Sociodemographic predictors of adherence to annual cervical cancer screening women. Cancer Nurs2000;23(5):350-6.

23. Borrayo E, Thomas J, Lawsin C. Cervical cancer screening among Latinas: the importance of referral and participation in parallel cancer screening behaviors. Womens Health2004;39(2):13-29.

24. Carter G. A study to determine factors that inhibit compliance with engaging in recommended Papanicolaou smear screening in African American women [dissertation]. Mississippi: University of Southern Mississippi 2008; 97p.

25. Chaudhry S, Fink A, Gelberg L, Brook R. Utilization of Papanicolaou smears by South Asian women living in the United States. J Gen Intern Med 2003;18(5):37784.

26. Lale N, Öry F, Detmar S: Factors associated with non-participation of Turkish women to cervical cancer screening in the Netherlands. [Abstract] Tijdschrift Sociale Geneeskunde 2003;81:184-8.

27. Fernandez-Esquer ME, Espinoza P, Ramirez AG, McAlister AL. Repeated Pap smear screening 
among Mexican-American women. Health Educ Res 2003;18(4):477-87.

28. Baranoski A, Horsburgh CR, Cupples LA, Aschengrau A, Stier E. Risk factors for non adherence with Pap testing in HIV-infected women. J Womens Health 2011;20(11):1635-43.

29. Cook N. A multi-levelapproach to understanding Pap smear compliance across community health centers in Florida. [dissertation]. Coral Gables:University of Miami 2008;94p.

30. Liao CC, Wang HY, Lin RS, Hsieh CY, Sung FC. Addressing Taiwan's high incidence of cervical cancer: factors associated with the Nation'slow compliance with Papanicolaou screening in Taiwan. Public Health 2006;120(12):1170-6.

31. Cook N, Kobetz E, Reis I, Fleming L, Martin L, Amofah $\mathrm{S}$. Role of patient race/ethnicity, insurance and age on Pap smear compliance across ten community health centers in Florida. Ethn Dis 2010;20(4):321-6.

32. Ramsey S, Cheadle A, Neighbor W, Gore E, Temple $\mathrm{P}$, Staiger T, Goldberg H. Relative impact of patient and clinic actors on adherence to primary care preventive serviceg uidelines: an exploratory study. Medical Care 2001;39(9):979-89.

33. Blackwell D, Martinez M, Getleman J. Women's compliance with public health guidelines for mammograms and Pap tests in Canada and United States. Womens Health Issues 2008;18:85-99.

34. Nguyen-Truong C. Lee-Lin F, Leo M, C., Gedaly-Duff V, Nail L, Wang P, Tran T. A community-based participatory research approach to understanding Pap testing adherence among Vietnamese American immigrants. JOGNN 2012;41(6):E26-40.

35. Nelson W, Moser R, Gaffey A, Waldron W. Adherence to cervical cancer screening guidelines for U.S. women aged 25-64: Data from the 2005 Health Information National Trends Survey (HINTS). J Womens Health 2009;18(11):1759-68.

36. Simoes E, Newschaffer C, Hagdrup N, Ali-Abarghoui F, Tao X, Mack N, Browson R. Predictors of compliance with recommended cervical cancer screening schedule: a population-based study. J Community Health 1999;24(2):115-30.

37. Gonzalez P, Castaneda S, Mills P, Talavera G, Elder J, Gallo L. Determinants of breast, cervical and colorectal cancer screening adherence in Mexican-American Women. J Community Health 2012;37(2):421-33.

38. De Alburquerque K, Frias $P$, de Andrade $C$, Aquino $E$, Menezes G, Szwarcwald C. Pap smear coverage and factors associated with non-participation in cervical cancer screening: an analysis of the cervical cancer prevention program in Pernambuco State, Brazil. Cad Saude Publica 2009;25(2):S301-9.

39. Matthews A, Brandenburg D, Johnson T, Hughes T. Correlates of under utilization of gynecological cancer screening among lesbian and heterosexual women. Prev Med2004;38(1):105-13.
40. Taylor V, Yasui Y, Burke N, Nguyen T, Acorda E, Thai $\mathrm{H}, \mathrm{Qu} \mathrm{P}$, Jackson JC. Pap testing adherence among Vietnamese American women. Cancer Epidemiol Biomarkers Prev 2004;13(4):613-9.

41. Augustson E, Vadaparampil S, Paltoo D, Kidd L, O'Malley A. Association between CBE, FOBT, and Pap smear adherence and mammography adherence among older low-income women. Prev Med 2003;36(6):734-9.

42. Williams J, Santoso J, Ling F, Przepiorka D. Pap smear non compliance among female obstetrics-gynecology residents. Gynecol Oncol 2003;90(3):597600.

43. Lazcano-Ponce E, Moss S, Cruz-Valdez A, Alonso P, Cesares-Queralt S, Martínez-León C, Hernández-Avila M. Factores que determinan la participación en el tamizaje de cáncer cervical en el estado de Morelos. Salud Pública Mex 1999;41(4):278-85.

44. Kahn J, Goodman E, Huang B, Slap G, Emans S. Predictors of Papanicolaou smear return in a hospitalbased adolescent and young adult clinic. Obstet Gynecol 2003;101(3):490-9.

45. MacLaughlan S, Lachance J, Gjelsvik A. Correlation between smoking status and cervical cancer screening: a cross-sectional study. J Low Genit Tract Dis 2011;15(2):114-19.

46. Baranowski T, Perry Ch, Parcel G. (2002). How individuals, environments, and health behavior interact. Social Cognitive Theory. In: Glanz K, Rimer B \& Lewis F (Eds.), Health behavior and health education. Theory, research and practice (pp. 160-184). San Francisco: Jossey Bass.

47. Nguyen AB, Hood KB, Belgrave FZ. The relationship between religiosity and cancer screening among Vietnamese women in the UnitedStates: the moderating role of acculturation. Womens Health2012;52(3):292-313.

48. Tung WC, Lu M, Cook D. Cervical cancer screening Taiwanese women: a transtheoretical approach. Oncol Nurs Forum 2010;37(4):E288-94.

49. Park S, Chang S, Chung C. Effects of a cognitionemotion focused program to increase public participation in Papanicolaou smear screening. Public Health Nurs 2005;22(4):289-98.

50. Egbert N, Parrott R. Self-efficacy and rural women's performance of breast and cervical cancer detection practices. J Health Commun 2011;6(3):219-33.

51. Hogenmiller J, Atwood J, Lindsey A, Johnson D, Hertzog M, Scott J. Self- Efficacy scale for Pap smear screening participation y sheltered women. Nurs Res 2007;56(6):369-77.

52. Fish J, Anthony D. UK lesbians and health care survey. Womens Health 2008;41(3):27-45.

53. De Araujo W, Alves M, de Oliveira B, Alves A, Faria L, do Nacimento L. Adherence of women aged 18 to 50 years to a cervical smear test in the Family Health Strategy. Rev Enferm 2012;7:15-22. 
54. Chen LS, Chou YJ, Tsay JH, Lee CH, Chou P, Huang $\mathrm{N}$. Variation in the cervical cancer screening compliance among women with disability. J Med Screen 2009;16(2):85-90.

55. Departamento de Estadísticas e Información en Sa- lud. Cobertura. Citado 8 Mayo 2013. Disponible en: http://www.deis.cl/cobertura/

56. Sociedad Chilena de Obstetricia y Ginecología. Noticias SOCHOG. Citado 8 Mayo 2013. Disponible en: http://www.sochog.cl/noticias-sochog.php?id=71. 